\title{
ON A THEOREM OF HURWITZ
}

\author{
by A. M. MACBEATH \\ (Received 23rd November, 1960)
}

1. Introduction. By a theorem of Hurwitz [3], an algebraic curve of genus $g \geqq 2$ cannot have more than $84(g-1)$ birational self-transformations, or, as we shall call them, automorphisms. The bound is attained for Klein's quartic

$$
x^{3} y+y^{3} z+z^{3} x=0
$$

of genus 3 [4]. In studying the problem whether there are any other curves for which the bound is attained, I was led to consider the universal covering space of the Riemann surface, which, as Siegel observed, relates Hurwitz's theorem to Siegel's own result [7] on the measure of the fundamental region of Fuchsian groups. Any curve with $84(g-1)$ automorphisms must be uniformized by a normal subgroup of the triangle group $(2,3,7)$, and, by a closer analysis of possible finite factor groups of $(2,3,7)$, purely algebraic methods yield an infinite family of curves with the maximum number of automorphisms. This will be shown in a later paper.

The aim of the present paper, however, is to show how one can prove the existence of such an infinite family without any knowledge of the algebraic structure of the triangle group. All one needs is a single curve with the maximum number of automorphisms-and for this we may use Klein's quartic. By lifting the group of automorphisms to the universal covering space we obtain an extension of the fundamental group of the curve. If we then collapse the fundamental group to the first homology group modulo $m$-which involves factoring out a normal subgroup of finite index-we arrive at a finite extension of the original group and a finite-sheeted covering space of the original Riemann surface. Thus, if $g$ is the genus of the original curve, we obtain, for every integer $m$, a curve with the maximum number of automorphisms and genus $(g-1) m^{2 g}+1$.

It is possible to construct an extension group of this kind for more general spaces, and the main part of the paper deals with this. The results belong, on the whole, to a familiar part of classical topology, but the present application requires a restatement of them which may be useful in other contexts.

In what follows, we shall not necessarily be interested in the whole group of homeomorphisms of a space, but rather in those which preserve some local structure, e.g. a differentiable or analytic structure. The term "automorphism" is used as an omnibus word to cover " homeomorphism", "differentiable homeomorphism of class $C^{r}$ ", or " one-one analytic mapping" according to context. We do not completely specify the kinds of local structure to which the results apply-perhaps there might be applications to local affine structures or local group structures, for instance. However, the immediate application we have in mind is that of analytic mappings of a Riemann surface.

2. Induced mappings on orbit space. Let $X$ be a connected topological space with some kind of local structure, e.g. a $C_{r}$-differentiable structure or a complex analytic structure. For any space $S$, let $A(S)$ denote the group of automorphisms of $S$, i.e. the group of homeo- 
morphisms of $S$ onto itself which preserve the local structure. Suppose that $N$ is a group of automorphisms of $X$ with the following property.

Each $x \in X$ has a neighbourhood $V(x)$ such that $V(x) \cap n V(x)=\emptyset$ if $1 \neq n \in N$.

It follows from $(A)$ that $N$ acts without fixed points. The $N$-orbits $\{n x: n \in N\}$ form the orbit space $X / N$ and the projection mapping $p: X \rightarrow X / N$, which maps each point $x$ on the orbit containing it, is a local homeomorphism, defining a local structure on $X / N$. An automorphism $f \in A(X)$ is called $N$-admissible if both $f$ and $f^{-1}$ map each $N$-orbit into an $N$-orbit; that is,

$$
x \in N y \text { if and only if } f x \in N f(y),
$$

or, in terms of the projection $p$,

$$
p x=p y \quad \text { if and only if }(p \circ f) x=(p \circ f) y .
$$

If $N x$ is an orbit, the $N$-admissible map $f$ maps $N x$ on another orbit $N y$, say, thus inducing a map $p^{*}(f)$ of the orbit-space $X / N$ onto itself. This map $p^{*}(f)$ is defined by the relation

$$
\left(p^{*}(f)\right)(N x)=N y=N f(x),
$$

or, in terms of the projection,

$$
p^{*}(f) \circ p=p \circ f
$$

Clearly $p^{*}(f) \circ p^{*}(g)=p^{*}(f \circ g), p^{*}(1)=1$, so that $p^{*}\left(f^{-1}\right)$ is a left and right inverse of $p^{*}(f)$. Thus $p^{*}(f)$ is an automorphism of $X / N$. The set of $N$-admissible automorphisms of $X$ thus forms a group, which we denote by $Z(X, N)$, and $p^{*}$ is a homomorphism from $Z(X, N)$ into $A(X / N)$. The following theorem gives the algebraic relationship between groups $Z(X, N)$ and $N$ within the larger group $A(X)$.

THEOREM 1. The group $Z(X, N)$ is the normalizer of $N$ in $A(X)$, and $N$ is the kernel of the projection homomorphism $p^{*}$.

Proof. We show first that $N$ is the kernel. Clearly $N \subset \operatorname{Ker} p^{*}$, so we need only show $\operatorname{Ker} p^{*} \subset N$. Let $f \in \operatorname{Ker} p^{*}$. Then, for each $x \in X, f x \in N x$; i.e., there is an $n \in N$, possibly varying from one point $x$ to another, such that $f x=n x$. For each $n \in N$, let $B(n)$ denote the set of $x$ such that $f x=n x$. Thus $X=\cup B(n)$, and, by $(A)$, the sets $B(n)$ are disjoint.

Let $x \in B(n)$ and choose a neighbourhood $W$ of $x$ so that (i) $W \subset V(x)$, (ii) $f(W) \subset n V(x)$. If $n \neq n^{\prime}, f(W) \cap n^{\prime} W \subset n V(x) \cap n^{\prime} V(x)=\emptyset$, by $(A)$. Thus $W$ does not meet any set $B\left(n^{\prime}\right)$ except $B(n)$, and $B(n)$ is open.

The connected space $X$ is thus the union of the disjoint open sets $B(n)$, and so precisely one of these sets, say $B\left(n_{0}\right)$, is non-empty. Thus $B\left(n_{0}\right)=X$ and $f=n_{0} \in N$.

To complete the proof, note first that the normalizer of $N$ is contained in $Z(X, N)$; for, if $t N=N t$, then $t N x=N t x$ and $t$ maps the orbit $N x$ onto the orbit $N t x$. Secondly, $Z(X, N)$ is contained in the normalizer, since $N$, being the kernel of the homomorphism $p^{*}$, is normal in $Z(X, N)$.

3. The universal covering space. Let $S$ be a connected, locally arc-connected, locally simply connected topological space. In this section we replace the space $X$ of $\S 2$ by the universal covering space $\tilde{S}$ of $S$, and we let $N$ denote the fundamental group of $S$ which can 
also be regarded as a discrete group of covering transformations of $\tilde{S}$. The orbit space $\tilde{S} / N$ can be identified with the original space $S$, and the projection $p$ is the usual projection in the theory of covering spaces $[1,2,5,6]$. It is convenient to recall the definition and basic properties of $\tilde{S}$.

A path in $S$ is a continuous mapping $C$ of the unit interval $I=[0,1]$ into $S$. The point $C(0)$ is called the initial point, $C(1)$ the end-point, and we speak of a path from $C(0)$ to $C(1)$. If $C, D$ are two paths such that $C(1)=D(0)$, we define $C D, C^{-1}$ as usual by the rules

$$
\begin{gathered}
C D(x)=C(2 x) \quad\left(0 \leqq x \leqq \frac{1}{2}\right), \quad C D(x)=D(2 x-1) \quad\left(\frac{1}{2} \leqq x \leqq 1\right), \\
C^{-1}(x)=C(1-x) .
\end{gathered}
$$

Two paths are considered equivalent if there is a homotopy from one to the other keeping the initial and end-points fixed. Let $S^{*}$ be the set of all path-classes.

If $c$ is the class of a path $C$ and $d$ is the class of a path $D$, we use $c d$ to denote the (welldefined) class of $C D$ and $c^{-1}$ to denote that of $C^{-1}$. If $f: S \rightarrow S$ is any continuous mapping and $C$ is a path, then $f \circ C$ is a path whose class depends only on the class of $C$. If $c$ is the class of $C, f * c$ denotes the class of $f \circ C$. Clearly

$$
f *(c d)=(f * c)(f * d), f * c^{-1}=(f * c)^{-1} \text {. }
$$

If $c$ is the class of a path $C$, define $p_{0}(c)=C(0), p_{1}(c)=C(1)$. Then we have also

$$
\begin{gathered}
p_{1}(c d)=p_{1}(d), \\
p_{i}(f * c)=f\left(p_{i}(c)\right) .
\end{gathered}
$$

Let $\mathbf{N}$ be a basis for the topology in $X$, consisting entirely of simply-connected open sets. Let $U, V \in \mathbf{N}$ and let $c$ be a path class from $x \in U$ to $y \in V$. For cuniz $z \in U$ define $d(z)$ to be the class of any path in $U$ from $z$ to $x$. Since $U$ is simply-connected, the class $d(z)$ depends only on z. Similarly, let $e(w)$, for $w \in V$, be the class of any path in $V$ from $y$ to $w$. Define $U c V \subset S^{*}$ to be the set of all classes

$$
\{d(z) c e(w): z \in U, w \in V\},
$$

and take the totality of sets $U c V$, where $U, V \in \mathbf{N}, c \in S^{*}, p_{0}(c) \in U, p_{1}(c) \in V$, as a basis for a topology in $S^{*}$.

Let $(\tilde{S}, x)$ be the subspace of $S^{*}$ consisting of all path classes with initial point $x$, i.e. $(\tilde{S}, x)=p_{0}^{-1}(x)$. Then $(\tilde{S}, x)$ is called the universal covering space of $S$ with base point $x$. If $h$ is a path class from $x$ to $y$, the left translation $\lambda_{h}\left(\lambda_{h}(c)=h c\right)$ maps $(\tilde{S}, y)$ into $(\tilde{S}, x)$. Since $h h^{-1}$ and $h^{-1} h$ are constant path-classes, $\lambda_{h}^{-1}=\lambda_{h^{-1}}$ and the two covering spaces are homeomorphic. Thus, for most purposes, $(\tilde{S}, x)$ and $(\tilde{S}, y)$ may be regarded as identical and denoted by $\tilde{S}$.

The closed path-classes which begin and end at $x$ form a group-the fundamental group $\pi_{1}(S, x)$. The left-translations

$$
\lambda_{c}: c \in \pi_{1}(S, x)
$$

form a discontinuous group $N$ of homeomorphisms of $(\tilde{S}, x)$. We shall call $N$ the Poincaré group. The Poincaré group and the fundamental group are isomorphic. 
If $p$ denotes the restriction of the mapping $p_{1}$ to $(\tilde{S}, x)$, then $p$ is a local homeomorphism, inducing a local structure on $(\tilde{S}, x)$ to correspond with that of $S$. The inverse image $p^{-1}(y)$ of any point $y \in S$ is an $N$-orbit; for if

$$
p(a)=p(b)=y,
$$

then $a b^{-1}$ is defined, $p\left(a b^{-1}\right)=x$, so $a b^{-1}=c \in \pi_{1}(S, x)$, and $a=\lambda_{c}(b) \in N b$. The points $y$ of $S$ are thus in one-one correspondence with the $N$-orbits $p^{-1}(y)$, the homeomorphisms of $N$ are automorphisms and it is easily verified that the correspondence between $N$-orbits and points of $S$ is a structure-preserving homeomorphism of $\widetilde{S} / N$ with $S$. Henceforth we drop the distinction between $\tilde{S} / N$ and $S$.

THEOREM 2. If $\tilde{S}$ is the universal covering space and $N$ the Poincaré group, then the projection homomorphism

is an epimorphism.

$$
p^{*}: Z(S, N) \rightarrow A(S)
$$

Proof. Let $f \in A(S)$. Let $h$ be a path class from $x$ to $f(x)$. If $c \in(\tilde{S}, x)$ then

$$
(f * c) \in(\tilde{S}, f(x))
$$

and the mapping $c \rightarrow f * c$ is a homeomorphism of $(\tilde{S}, x)$ onto $(\tilde{S}, f(x))$. Define the automorphism

$$
\tilde{f} \in A(\tilde{S}, x)
$$

by the relation $f(c)=h(f * c)$. By (4) and (5),

$$
p(\tilde{f}(c))=p(\tilde{f} * c)=f(p(c)) \text {. }
$$

It follows that, if $p(c)=p(d)$, then $p(f(c))=p(f(d))$, with a similar result for $f^{-1}$, since $f^{-1}(c)=h^{\prime}\left(f^{-1} * c\right)$, where $h^{\prime}=f^{-1} * h^{-1}$. Thus, by (1), $f \in Z(S, N)$. Finally, (2) and (6) show that $f=p^{*}(\tilde{f})$, which proves Theorem 2 .

Now let us consider the action of the automorphism $f \in A(S)$ on a closed path class $c \in \pi_{1}(S, x)$. The automorphism $f$ maps $c$ into $f * c \in \pi_{1}(S, f(x))$. To identify the two fundamental groups $\pi_{1}(S, x)$ and $\pi_{1}(S, f(x))$ we need a path class $h$ from $x$ to $f(x)$ and we choose this to be the same $h$ that was chosen in the proof of Theorem 2. The pair $(f, h)$ induces the group automorphism of $\pi_{1}(S, x)$ :

$$
c \rightarrow c^{\prime}=h(f * c) h^{-1} \text {. }
$$

Now consider the relation between the corresponding elements $\lambda_{c}, \lambda_{c^{\prime}}$ of the Poincaré group. From the relation in $(\tilde{S}, x)$,

we deduce, by (3), in $(\tilde{S}, f(x))$

$$
b=\lambda_{c}(a)=c a
$$

$$
f * b=(f * c)(f * a)
$$

To return to $(S, x)$ we need the path $h$, and derive

or

$$
h(f * b)=h(f * c) h^{-1} h(f * a)
$$

$$
f^{x}(b)=c^{\prime} f^{x}(a)
$$


Since $b=\lambda_{c}(a)$, and $a$ is arbitrary,

or

$$
\tilde{f} \circ \lambda_{c}=\lambda_{c^{\prime}} \circ \tilde{f}
$$

We have thus proved

$$
\lambda_{c^{\prime}}=\tilde{f} \circ \lambda_{c} \circ f^{-1} \text {. }
$$

THEOREM 3. A group automorphism of $\pi_{1}(S)$ induced by an automorphism $f$ of $S$ is the same as the group automorphism of $N$ obtained by transformation with a certain $\tilde{f} \in p^{*-1}(f) \subset Z(\tilde{S}, N)$.

4. Regular covering spaces. Let $S_{1}$ be a connected, locally arc-connected, locally simplyconnected space, and let $N_{1}$ be a group of automorphisms of $S_{1}$ satisfying condition $(A)$. If there is a structure-preserving homeomorphism of $S_{1} / N_{1}$ onto $S$, then $S_{1}$ is called a regular covering of $S$.

Regular coverings of $S$ can be obtained from the universal covering spaces $\widetilde{S}$ as follows. Let $M$ be a normal subgroup of the Poincaré group $N$. Then, as in the proof of Theorem 1, $N_{1}=N / M$ acts as a group of transformations of $S_{1}=\tilde{S} / M$, the orbit space $S_{1} / N_{1}$ being identifiable with $\tilde{S} / N=S$. It can be shown that every regular covering space of $S$ can be obtained in this way, and that any regular covering space of $S$ which is simply-connected can be mapped one-to-one onto $\tilde{S}$ by a homeomorphism which commutes with the projection operator. See e.g. [2], [6].

If the group $N_{1}$ has a finite number $k$ of elements, each point of $S$ will correspond to $k$ points of $S_{1}$ and $S_{1}$ is called a $k$-sheeted regular covering. In this case, $k$ is the index of $M$ in $N$. If $S$ is triangulable, then to any fine enough triangulation of $S$ corresponds a triangulation of $S_{1}$ in which there are exactly $k$ times as many simplexes of each dimension. Thus, if $\chi$ benotes the Euler characteristic, we have

$$
\chi\left(S_{1}\right)=k \chi(S) .
$$

Let the projections and their associated homomorphisms be denoted by

$$
\begin{array}{lll}
p: \tilde{S} \rightarrow S, & r: \tilde{S} \rightarrow S_{1}, & q: S_{1} \rightarrow S, \\
p^{*}: Z(\tilde{S}, N) \rightarrow A(S), & r^{*}: Z(\tilde{S}, M) \rightarrow A\left(S_{1}\right), & q^{*}: Z\left(S_{1}, N_{1}\right) \rightarrow A(S) .
\end{array}
$$

Then $p=q \circ r, p$ and $r$ are onto, $N \subset Z(\widetilde{S}, M)$ (since $M$ is normal in $N$ ) and $r^{*}(N)=N_{1}$.

We shall call $S_{1}$ a full covering space of $S$ if $q^{*}$ is an epimorphism. Thus the universal covering space is a full covering space.

THEOREM 4. $\tilde{S} / M$ is a full covering space of $S$ if and only if

$$
Z(\tilde{S}, N) \subset Z(\tilde{S}, M) \text {. }
$$

From Theorem 1, we deduce

CoRollary 1. $\bar{S} / M$ is a full covering space of $S$ if and only if $M$ is normal in $Z(\tilde{S}, N)$.

From Theorem 3, we deduce

CoRollary 2. $\tilde{S} / M$ is a full covering space of $S$ if and only if $M$ is invariant under all the group automorphisms of $N$ induced by transformations of $A(S)$.

CoROLlary 3. If $M$ is a characteristic subgroup of $N$, then $\tilde{S} / M$ is a full covering.

In the proof of Theorem 4, we require the following lemma. 
Lemma 1. If $f \in Z(\tilde{S}, M)$ and $r^{*}(f) \in Z\left(S_{1}, N_{1}\right)$, then $p^{*}(f)=\left(q^{*} \circ r^{*}\right)(f)$. (cf. (2))

Proof. Since $r^{*}(f) \in Z\left(S_{1}, N_{1}\right)$, it follows that $\left(q^{*} \circ r^{*}\right)(f)$ is defined. From the formulae

$$
(r \circ f)(x)=\left(r^{*}(f) \circ r\right)(x), \quad\left(q \circ f_{1}\right)(y)=\left(q^{*}\left(f_{1}\right) \circ q\right)(y),
$$

it follows, on putting $f_{1}=r^{*}(f), y=r(x)$, that

$$
(q \circ r \circ f)(x)=q \circ\left(r^{*}(f) \circ r\right)(x)=\left(q \circ f_{1}\right)(y)=\left(q^{*}\left(f_{1}\right) \circ q\right)(y)=\left(q^{*} \circ r^{*}\right)(f) \circ(q \circ r) x .
$$

That is

$$
(p \circ f) x=\left(q^{*} \circ r^{*}\right)(f) \circ p(x) .
$$

An exactly similar argument applies to $f^{-1}$. On comparing (8) with the defining property (2) of $p^{*}(f)$, we deduce that $p^{*}(f)=\left(q^{*} \circ r^{*}\right)(f)$.

Proof of Theorem 4. (i) First suppose that $Z(\tilde{S}, N) \subset Z(\tilde{S}, M)$. If $f \in A(S)$, then, by Theorem 2, $f=p^{*}(\tilde{f})$ where $\tilde{f} \in Z(\tilde{S}, N)$, so that (Theorem 1) $\tilde{f} N=N f^{*}$. Apply the homomorphism $r$. Then $r^{*}(\tilde{f}) N_{1}=N_{1} r^{*}(\tilde{f})$. Thus $r^{*}(\tilde{f}) \in Z\left(S_{1}, N_{1}\right)$. Hence by Lemma 1 , since $\tilde{f} \in Z(\tilde{S}, M), f=p^{*}(\tilde{f})=\left(q^{*} \circ r^{*}\right)(\tilde{f}) \in q^{*}\left(Z\left(S_{1}, N_{1}\right)\right)$, and $q^{*}$ is an epimorphism.

(ii) Conversely, suppose that $q^{*}$ is an epimorphism, so that, for each $\tilde{f} \in Z(\tilde{S}, N)$, there exists $f_{1} \in Z\left(S_{1}, N_{1}\right)$ such that $p^{*}(\tilde{f})=q^{*}\left(f_{1}\right)$. Now $\tilde{S}$, being simply-connected, is the universal covering space of $S_{1}$, and so, by Theorem 2 ,

$$
f_{1}=r^{*}\left(f_{2}\right), \quad f_{2} \in Z(\widetilde{S}, M) \text {. }
$$

Then, by Lemma $1, p^{*}(\tilde{f})=q^{*}\left(f_{1}\right)=\left(q^{*} \circ r^{*}\right)\left(f_{2}\right)=p^{*}\left(f_{2}\right)$. Hence $\tilde{f} \in f_{2} N$. But $f_{2} \in Z(\tilde{S}, M)$ and $N \subset Z(\tilde{S}, M)$, since $M$ is normal in $N$. Hence $\tilde{f} \in Z(\tilde{S}, M)$ and $Z(\tilde{S}, N) \subset Z(\tilde{S}, M)$.

5. Analytic manifolds. Let $S$ be a compact complex analytic manifold and suppose that the first homology group with integer coefficients $H_{1}(S, Z)$ has positive rank. Suppose also that the group $A(S)$ is known to be finite. Let $N$ denote the Poincare group, so that $N$ is finitely generated and $H_{1}(S, Z) \simeq N /[N, N]$. The commutator subgroup $[N, N]$ is a characteristic subgroup.

Similarly, if $m$ is any integer, the Burnside $m$-kernel $\left\{N^{m}\right\}$ generated by the $m$ th powers of all elements of $N$ is a characteristic subgroup. Hence the product $M(m)=\left\{N^{m}\right\}[N, N]$ is also a characteristic subgroup and the factor group

$$
N_{1}(m)=N / M(m)
$$

is finite, being a finitely generated abelian group in which each $m$ th power is equal to unity. However, the order $t(m)$ is at least equal to $m^{R_{1}}$, where $R_{1}$ is the first Betti number, so that $t(m)$ tends to infinity with $m$.

Let $S_{1}(m)=\tilde{S} / M(m)$. Since $M(m)$ is a characteristic subgroup, by Theorem 4, Corollary 3, $S_{1}(m)$ is a full covering of $S$ with $t(m)$ sheets. Hence, dropping the $m$ for convenience, we deduce that $Z\left(S_{1}, N_{1}\right) / N_{1} \simeq A(S)$. The order of $Z\left(S_{1}, N_{1}\right)$ is $t(m)$ times the order of $A(S)$, and the order of $A\left(S_{1}\right)$ is at least as great as that of the subgroup $Z\left(S_{1}, N_{1}\right)$. We have shown:

If the compact analytic manifold $S$, with positive first Betti number, admits a group $A(S)$ of $n$ automorphisms, then, for infinitely many values of the integer $k$, there is a $k$-sheeted regular covering space of $S$ with at least kn automorphisms. 
6. Application to algebraic curves. Let $S$ be the Riemann surface of an algebraic curve, genus $g \geqq 2$. Suppose that the number of automorphisms of $S$ is $h(g-1)$, where $h$ is a rational number whose denominator is a factor of $g-1$.

The Poincaré group of $S[6, \mathrm{p} .170]$ is defined by $2 g$ generators $a_{1}, b_{1}, \ldots, a_{\theta}, b_{\theta}$, and the relation

$$
\prod_{v=1}^{g}\left[a_{v}, b_{v}\right]=1 .
$$

If, as in $\S 5$, we form the product of the commutator subgroup and the Burnside kernel, the resulting factor group $N_{1}(m)$ is a product $Z_{m}^{2 g}$ of $2 g$ cyclic groups of order $m$. The corresponding covering space $S_{1}$ will have genus $g_{1}$ given by

$$
g_{1}-1=m^{2 g}(g-1) \text {, }
$$

by (7), since $\chi\left(S_{1}\right)=2-2 g_{1}, \chi(S)=2-2 g$. Hence we have proved

THEOREM 6. If there is a curve of genus $g \geqq 2$ with a group of $h(g-1)$ birational selftransformations, then there is a curve of genus $g_{1}$ with $h\left(g_{1}-1\right)$ birational self-transformations, where

$$
g_{1}=m^{2 g}(g-1)+1
$$

and $m$ is any positive integer.

COROLLARY. If we take $S$ to be Klein's quartic of genus 3 with 168 birational self-transformations, we deduce that, for any integer $m$, there is a curve of genus $2 m^{6}+1$ with $84(g-1)$ birational self-transformations.*

Thus Hurwitz's bound $84(g-1)$ is attained for infinitely many values of $g$.

$\mathrm{I}$ am indebted to $\mathrm{Dr} \mathrm{I}$. T. Adamson for having read through the first draft of this paper and making several useful suggestions.

\section{REFERENCES}

1. C. Chevalley, Theory of Lie groups I (Princeton, 1946), 50-56.

2. S. T. Hu, Homotopy theory, (New York, 1959), 93-97.

3. A. Hurwitz, Uber algebraische Gebilde mit eindeutigen Transformationen in sich, Math. Ann. 41 (1893), 403-442.

4. F. Klein, Uber die Transformation siebenter Ordnung der elliptischen Funktionen, Math. Ann. 14 (1879), 428-471.

5. L. S. Pontrjagin, Topological groups (Princeton, 1946), Chapter VIII.

6. H. Siefert and W. Threlfall, Lehrbuch der Topologie (Leipzig, 1934), Chapter VIII.

7. C. L. Siegel, Some remarks on discontinuous groups, Ann. of Math., 46 (1945), 708-718.

\section{QUEEN'S COLLEGE}

\section{DUNDEE}

* Note added in proof. The group corresponding to the curve of least genus found above $(g=129, m=2)$ turns out to be isomorphic to the group $(8,7 \mid 2,3)$ considered by Leech and Mennicke (these Proceedings 5 (1961), 25-29). 